\title{
Inequalities for low-energy symmetric nuclear matter
}

\author{
Dean Lee \\ North Carolina State University, Department of Physics, Raleigh, NC 27695, USA
}

\begin{abstract}
Using effective field theory we prove inequalities for the correlations of two-nucleon operators in low-energy symmetric nuclear matter. For physical values of operator coefficients in the effective Lagrangian, the $S=1, I=0$ channel correlations must have the lowest energy and longest correlation length in the two-nucleon sector. This result is valid at nonzero density and temperature.

PACS numbers: 13.75.Cs, 21.30.-x, 21.65.+f,
\end{abstract}

Keywords: Inequalities, nuclear matter, effective field theory, lattice, deuteron 
The study of inequalities in quantum chromodynamics (QCD) dates back over twenty years. Even though the information one may extract from these inequalities is limited, they provide rare insights into the nonperturbative QCD vacuum [1] [2] [3] and hadronic spectrum [4] [5] [6] [7] which currently cannot be addressed by other purely analytic methods. Most rigorous QCD inequalities are derived using the Euclidean functional integral. The close relationship to lattice QCD simulations should be apparent. Typically one compares the magnitude of various observable quantities by means of the Cauchy-Schwarz or Hölder inequalities. The comparison requires positivity of the functional integral measure for at least one of the quantities being compared and in some cases positivity of the observable for arbitrary background gauge configurations. These same attributes are also important in lattice QCD in connection with the well-known sign/phase problem. A review of the literature on QCD inequalities can be found in [8].

Recently there has been some interest in simulations of nuclear matter on the lattice using effective field theory 9] 10 11] 12]. These are low-energy simulations with nucleons and sometimes pions as point particles rather than quarks and gluons. Motivated by these nonperturbative studies, we consider here the subject of effective field theory inequalities for nuclear matter. Our focus and application will be to nuclear matter, but much of the interesting physics is of a universal nature and appears in other systems such as trapped Fermi gases near a Feshbach resonance [13] 14].

It should be obvious that effective field theory does not constrain properties of the vacuum, nucleon and pion masses, or other few-body spectra at zero chemical potential and temperature. These quantities are usually phenomenological inputs which are used to determine the coefficients of operators in the effective Lagrangian. Hence the inequalities which we prove here concern correlation functions at nonzero chemical potential and/or temperature. The results are most interesting at nucleon densities where the average nucleon separation is less than the scattering length, and the physics is strongly coupled.

We let $N$ represent the nucleon fields,

$$
N=\left[\begin{array}{l}
p \\
n
\end{array}\right] \otimes\left[\begin{array}{l}
\uparrow \\
\downarrow
\end{array}\right]
$$

We use $p(n)$ to represent protons(neutrons) and $\uparrow(\downarrow)$ to represent up(down) spins. We use $\vec{\tau}$ to represent Pauli matrices acting in isospin space and $\vec{\sigma}$ to represent Pauli matrices 
acting in spin space. We assume exact isospin symmetry. In the non-relativistic limit and below the threshold for pion production, we can write the lowest order terms in the effective Lagrangian [15] 16] 17] [18] [19] 20] in several equivalent ways. Two of the possibilities are

$$
\begin{aligned}
& \mathcal{L}=\bar{N}\left[i \partial_{0}+\frac{\vec{\nabla}^{2}}{2 m_{N}}-\left(m_{N}^{0}-\mu\right)\right] N-\frac{1}{2} C_{S} \bar{N} N \bar{N} N-\frac{1}{2} C_{T} \bar{N} \vec{\sigma} N \cdot \bar{N} \vec{\sigma} N, \\
& \mathcal{L}=\bar{N}\left[i \partial_{0}+\frac{\vec{\nabla}^{2}}{2 m_{N}}-\left(m_{N}^{0 \prime}-\mu\right)\right] N-\frac{1}{2} C_{S}^{\prime} \bar{N} N \bar{N} N-\frac{1}{2} C_{U}^{\prime} \bar{N} \vec{\tau} N \cdot \bar{N} \vec{\tau} N .
\end{aligned}
$$

At this stage we leave out three-nucleon forces, but we will comment on this later in our discussion. We assume that the high-momentum modes have been removed by some regularization procedure though details are unimportant in this discussion.

Consider states with two nucleons at the same point. In order to maintain overall antisymmetry, we can have either a spin $S=0$, isospin $I=1$ state, or an $S=1, I=0$ state. These two states are eigenstates of the various interaction operators, and the corresponding eigenvalues are shown in Table 1. At lowest order in the pionless effective theory, we do not have mixing between $S$ and $D$ waves in the $S=1, I=0$ channel.

Table 1: Eigenvalues of interaction operators

\begin{tabular}{|l|l|l|}
\hline & $S=1, I=0$ & $S=0, I=1$ \\
\hline$\frac{1}{2}: \bar{N} N \bar{N} N:$ & 1 & 1 \\
\hline$\frac{1}{2}: \bar{N} \vec{\sigma} N \cdot \bar{N} \vec{\sigma} N:$ & 1 & -3 \\
\hline$\frac{1}{2}: \bar{N} \vec{\tau} N \cdot \bar{N} \vec{\tau} N:$ & -3 & 1 \\
\hline
\end{tabular}

Clearly

$$
\begin{gathered}
: \bar{N} N \bar{N} N:=-\frac{1}{2}: \bar{N} \vec{\sigma} N \cdot \bar{N} \vec{\sigma} N:-\frac{1}{2}: \bar{N} \vec{\tau} N \cdot \bar{N} \vec{\tau} N: \\
C_{U}^{\prime}=-C_{T}, \quad C_{S}^{\prime}=C_{S}-2 C_{T}
\end{gathered}
$$

In the real world two-nucleon low-energy scattering is attractive in both the $S=1, I=0$ and $S=0, I=1$ channels. It is also more strongly attractive in the $S=1, I=0$ channel. From this we deduce that

$$
\begin{aligned}
& C_{S}<3 C_{T}, \quad C_{T}<0, \\
& C_{S}^{\prime}<-C_{U}^{\prime}, \quad C_{U}^{\prime}>0 .
\end{aligned}
$$


These inequalities can be verified nonperturbatively by solving the Schrodinger equation and determining phase shifts [11] or by summing all bubble diagrams [21]. The analysis is straightforward since we have only a simple contact potential.

The grand canonical partition function is given by

$$
Z_{G} \propto \int D N D \bar{N} \exp \left(-S_{E}\right)=\int D N D \bar{N} \exp \left(\int d^{4} x \mathcal{L}_{E}\right),
$$

where we choose to write $\mathcal{L}_{E}$ as

$$
\mathcal{L}_{E}=-\bar{N}\left[\partial_{4}-\frac{\vec{\nabla}^{2}}{2 m_{N}}+\left(m_{N}^{0 \prime}-\mu\right)\right] N-\frac{1}{2} C_{S}^{\prime} \bar{N} N \bar{N} N-\frac{1}{2} C_{U}^{\prime} \bar{N} \vec{\tau} N \cdot \bar{N} \vec{\tau} N
$$

Using Hubbard-Stratonovich transformations [22] 23], we can rewrite $Z_{G}$ as

$$
Z_{G} \propto \int D N D \bar{N} D f D \vec{\phi} \exp \left(\int d^{4} x \mathcal{L}_{E}^{f, \vec{\phi}}\right)
$$

where

$$
\mathcal{L}_{E}^{f, \vec{\phi}}=-\bar{N}\left[\partial_{4}-\frac{\vec{\nabla}^{2}}{2 m_{N}}+\left(m_{N}^{0 \prime}-\mu\right)\right] N+C_{S}^{\prime} f \bar{N} N+i C_{U}^{\prime} \vec{\phi} \cdot \bar{N} \vec{\tau} N-\mathcal{V}(f, \vec{\phi})
$$

and

$$
-\mathcal{V}(f, \vec{\phi})=\frac{1}{2} C_{S}^{\prime} f^{2}-\frac{1}{2} C_{U}^{\prime} \vec{\phi} \cdot \vec{\phi}
$$

We note that $C_{S}^{\prime}<0$ and $C_{U}^{\prime}>0$, and so the $f$ and $\vec{\phi}$ integrations are convergent.

Let $M$ be the nucleon matrix generated by the background Hubbard-Stratonovich fields,

$$
M=-\left[\partial_{4}-\frac{\vec{\nabla}^{2}}{2 m_{N}}+\left(m_{N}^{0 \prime}-\mu\right)\right]+C_{S}^{\prime} f+i C_{U}^{\prime} \vec{\phi} \cdot \vec{\tau}
$$

We note that

$$
\tau_{2} M \tau_{2}=M^{*}
$$

where $M^{*}$ is the complex conjugate of $M$, not the Hermitian adjoint. We conclude that $\operatorname{det} M$ must be real. Since $M$ does not couple up and down spins we can write $M$ with two identical diagonal blocks,

$$
M=\left[\begin{array}{cc}
M_{\uparrow} & 0 \\
0 & M_{\downarrow}
\end{array}\right], \quad M_{\uparrow}=M_{\downarrow} .
$$

We note that $\operatorname{det} M_{\uparrow}$ must also be real, and so

$$
\operatorname{det} M=\operatorname{det} M_{\uparrow} \operatorname{det} M_{\downarrow}=\left(\operatorname{det} M_{\uparrow}\right)^{2} \geq 0 .
$$


Although not relevant to the main line of this discussion, we point out that actually $\operatorname{det} M_{\uparrow} \geq$ 0. This is because $\tau_{2}$ is antisymmetric and therefore the real eigenvalues of $M_{\uparrow}$ are doubly degenerate [27].

We note the similarity between (15) and the relation

$$
\gamma_{5} M \gamma_{5}=M^{\dagger}
$$

for the quark matrix in QCD at zero chemical potential. From (18) one can show positivity of the quark matrix determinant for two degenerate flavors and the dominance of pion correlation functions [24]. In our case, however, we observe that (15I) is also valid at nonzero chemical potential.

Let us now consider the two-nucleon operator

$$
A(x)=\left[N_{\uparrow}\right]_{i}\left[\tau_{2}\right]_{i j}\left[N_{\downarrow}\right]_{j}(x)
$$

where $N_{\uparrow}$ and $N_{\downarrow}$ are up and down spin projections of $N$. Since $A$ has quantum number $I=0$, overall antisymmetry requires it also have quantum number $S=1$. The two-point correlation function for $A$ is

$$
\left\langle A(x) A^{\dagger}(0)\right\rangle_{\mu, T}=\left\langle\left[N_{\uparrow}\right]_{i}\left[\tau_{2}\right]_{i j}\left[N_{\downarrow}\right]_{j}(x)\left[N_{\downarrow}^{*}\right]_{l}\left[\tau_{2}\right]_{l m}\left[N_{\uparrow}^{*}\right]_{m}(0)\right\rangle_{\mu, T} .
$$

Using our Euclidean functional integral representation, we have

$$
\left\langle A(x) A^{\dagger}(0)\right\rangle_{\mu, T}=\int D \Theta\left[M_{\downarrow}^{-1}(x, 0)\right]_{j l}\left[\tau_{2}\right]_{l m}\left[M_{\uparrow}^{-1}(x, 0)\right]_{i m}\left[\tau_{2}\right]_{i j},
$$

where $D \Theta$ is the positive normalized measure defined by

$$
D \Theta=\frac{D f D \vec{\phi} \operatorname{det} M \exp \left(-\int d^{4} x \mathcal{V}(f, \vec{\phi})\right)}{\int D f D \vec{\phi} \operatorname{det} M \exp \left(-\int d^{4} x \mathcal{V}(f, \vec{\phi})\right)}
$$

From the symmetry properties of $\tau_{2}$ under transposition and (15),

$$
\begin{aligned}
{\left[M_{\downarrow}^{-1}(x, 0)\right]_{j l}\left[\tau_{2}\right]_{l m}\left[M_{\uparrow}^{-1}(x, 0)\right]_{i m}\left[\tau_{2}\right]_{i j} } & =\left[M_{\downarrow}^{-1}(x, 0)\right]_{j l}\left[\tau_{2}\right]_{j i}\left[M_{\uparrow}^{-1}(x, 0)\right]_{i m}\left[\tau_{2}\right]_{m l} \\
& =\left[M_{\downarrow}^{-1}(x, 0)\right]_{j l}\left[M_{\uparrow}^{-1}(x, 0)\right]_{j l}^{*} \\
& =\sum_{j l}\left|\left[M_{\uparrow}^{-1}(x, 0)\right]_{j l}\right|^{2} .
\end{aligned}
$$

Thus

$$
\left\langle A(x) A^{\dagger}(0)\right\rangle_{\mu, T}=\int D \Theta \sum_{j l}\left|\left[M_{\uparrow}^{-1}(x, 0)\right]_{j l}\right|^{2} .
$$


Consider now the more general operator

$$
B(x)=\left[N_{\uparrow}\right]_{i}[b]_{i j}\left[N_{\downarrow}\right]_{j}(x)
$$

for arbitrary $2 \times 2$ matrix $[b]_{i j}$. We find

$$
\left\langle B(x) B^{\dagger}(0)\right\rangle_{\mu, T}=\int D \Theta G_{B}(x),
$$

where

$$
G_{B}(x)=\left[M_{\downarrow}^{-1}(x, 0)\right]_{j l}[b]_{m l}^{*}\left[M_{\uparrow}^{-1}(x, 0)\right]_{i m}[b]_{i j} .
$$

From the Cauchy-Schwarz inequality,

$$
\begin{aligned}
\left|G_{B}(x)\right| & \leq \sqrt{\sum_{i j l m}\left|\left[M_{\uparrow}^{-1}(x, 0)\right]_{i m}\right|^{2}\left|\left[M_{\downarrow}^{-1}(x, 0)\right]_{j l}\right|^{2}} \sqrt{\sum_{i^{\prime} j^{\prime} l^{\prime} m^{\prime}}\left|[b]_{i^{\prime} j^{\prime}}\right|^{2}\left|[b]_{m^{\prime} l^{\prime}}\right|^{2}} \\
& \leq \sum_{i m}\left|\left[M_{\uparrow}^{-1}(x, 0)\right]_{i m}\right|^{2} \sum_{j k}\left|[b]_{j k}\right|^{2} .
\end{aligned}
$$

We conclude that

$$
\left|\left\langle B(x) B^{\dagger}(0)\right\rangle_{\mu, T}\right| \leq \int D \Theta\left|G_{B}(x)\right| \leq \gamma\left\langle A(x) A^{\dagger}(0)\right\rangle_{\mu, T}
$$

where

$$
\gamma=\sum_{j k}\left|[b]_{j k}\right|^{2}
$$

Let us take the zero temperature limit at fixed nonzero nucleon density. This will require tuning the chemical potential $\mu$ to match the desired density as we lower the temperature. If we take the separation $x \rightarrow \infty$ in the Euclidean time direction, we deduce from (29) that the energy of the lowest state with the quantum numbers of $A$ (i.e., $S=1, I=0$ ) must be less than or equal to the lowest state with the quantum numbers of $B$,

$$
E_{A}^{0} \leq E_{B}^{0}
$$

This result was known from the outset for zero density. The non-trivial result is that it also holds true at nonzero density. In particular if we are in a region of the phase diagram where the deuteron is unbound, then all states with the quantum numbers of $B$ must also be unbound. A formula due to Lüscher [25] tells us that in a periodic box of length $L$, the energy of the lowest two-particle scattering state relative to threshold is

$$
E_{0}=\frac{4 \pi a}{m_{N} L^{3}}\left[1+O\left(a L^{-1}\right)\right]
$$


where is $a$ is the scattering length with sign convention,

$$
k \cot \delta_{0}=-\frac{1}{a}+\frac{1}{2} r_{0} k^{2}+\ldots
$$

By combining (31) and (32), we find in the unbound case the following inequality for the scattering lengths [28],

$$
a_{A} \leq a_{B}
$$

If we take the limit $x \rightarrow \infty$ in any spatial direction, we find that the correlation length for $A$ must be greater than or equal to the correlation length for $B$,

$$
\xi_{A} \geq \xi_{B}
$$

This holds true for any density and any temperature. In particular if $\xi_{B}$ diverges, as would occur in a superfluid phase transition, then $\xi_{A}$ must also diverge. In that case one can use (29) and (30) to bound the relative sizes of the dinucleon condensates.

The inequalities (31), (34), and (35) were proven for operators $B$ of the specific form given in (25). It is straightforward to generalize to the case when the nucleons have arbitrary spin directions and/or are not at the same point. Let

$$
B_{b, y}(x)=[N(x+y)]_{i}[b]_{i j}[N(x-y)]_{j},
$$

where $[b]_{i j}$ is a general $4 \times 4$ matrix. One can show for some positive parameters $\gamma_{1}, \gamma_{2}, \gamma_{3}$, $\gamma_{4}$,

$$
\begin{aligned}
\left|\left\langle B_{b^{\prime}, y^{\prime}}(x) B_{b, y}^{\dagger}(0)\right\rangle_{\mu, T}\right| & \leq \gamma_{1}\left\langle A\left(x-y^{\prime}\right) A^{\dagger}(-y)\right\rangle+\gamma_{2}\left\langle A\left(x-y^{\prime}\right) A^{\dagger}(y)\right\rangle \\
& +\gamma_{3}\left\langle A\left(x+y^{\prime}\right) A^{\dagger}(-y)\right\rangle+\gamma_{4}\left\langle A\left(x+y^{\prime}\right) A^{\dagger}(y)\right\rangle .
\end{aligned}
$$

From (37) we conclude that the inequalities (31), (34), and (35) hold for any two-nucleon operator $B$,

$$
B(x)=\int_{\Omega} d^{4} y[N(x+y)]_{i}[b(y)]_{i j}[N(x-y)]_{j},
$$

where the region of integration $\Omega$ is bounded.

In summary we have shown that the $S=1, I=0$ channel must have the lowest energy and longest correlation length in the two-nucleon sector. These results are valid at arbitrary density and temperature and can be readily checked in lattice simulations using chiral 
effective field theory. Assuming that the effective Lagrangian can describe the relevant physics, it would be interesting to study two-nucleon correlations near the region of the phase diagram where the deuteron becomes unbound. In principle these results can also be checked in lattice QCD at finite density, though the complex phase problem will make the calculation exceedingly difficult.

It turns out that the addition of the three-nucleon force term $(\bar{N} N)^{3}$ [26] can be introduced by modifying the coupling of $f$ and $\bar{N} N$ while preserving positivity of $\operatorname{det} M$ and the conjugate similarity relation in (15). Other modifications can also be made for various higher order terms in the effective Lagrangian. These and other extensions will be discussed in future work.

The author thanks Paulo Bedaque, Jiunn-Wei Chen, and Thomas Schaefer for several helpful discussions. He also thanks Paulo for organizing the Summer of Lattice Workshop 2004 at Lawrence Berkeley Laboratory where part of this research was completed. This work was supported by the Department of Energy.

[1] C. Vafa and E. Witten, Nucl. Phys. B234, 173 (1984).

[2] C. Vafa and E. Witten, Phys. Rev. Lett. 53, 535 (1984).

[3] C. Vafa and E. Witten, Commun. Math. Phys. 95, 257 (1984).

[4] E. Witten, Phys. Rev. Lett. 51, 2351 (1983).

[5] S. Nussinov, Phys. Rev. Lett. 51, 2081 (1983).

[6] S. Nussinov, (2003), hep-ph/0306187.

[7] T. D. Cohen, Phys. Rev. Lett. 91, 032002 (2003), hep-ph/0304024.

[8] S. Nussinov and M. A. Lampert, Phys. Rept. 362, 193 (2002), hep-ph/9911532.

[9] H. M. Müller, S. E. Koonin, R. Seki, and U. van Kolck, Phys. Rev. C61, 044320 (2000), nucl-th/9910038.

[10] J.-W. Chen and D. B. Kaplan, (2003), hep-lat/0308016.

[11] D. Lee, B. Borasoy, and T. Schafer, (2004), nucl-th/0402072.

[12] T. Abe, R. Seki, and A. N. Kocharian, (2003), nucl-th/0312125.

[13] J. Kinast, S. L. Hemmer, M. E. Gehm, A. Turlapov, and J. E. Thomas, Phys. Rev. Lett. 92, $150402(2004)$. 
[14] C. A. Regal, M. Greiner, and D. S. Jin, Phys. Rev. Lett. 92, 040403 (2004).

[15] S. Weinberg, Phys. Lett. B251, 288 (1990).

[16] S. Weinberg, Nucl. Phys. B363, 3 (1991).

[17] S. Weinberg, Phys. Lett. B295, 114 (1992), hep-ph/9209257.

[18] D. B. Kaplan, M. J. Savage, and M. B. Wise, Nucl. Phys. B478, 629 (1996), nucl-th/9605002.

[19] D. B. Kaplan, M. J. Savage, and M. B. Wise, Phys. Lett. B424, 390 (1998), nucl-th/9801034.

[20] D. B. Kaplan, M. J. Savage, and M. B. Wise, Nucl. Phys. B534, 329 (1998), nucl-th/9802075.

[21] S. R. Beane, P. F. Bedaque, A. Parreno, and M. J. Savage, (2003), hep-lat/0312004.

[22] R. L. Stratonovich, Soviet Phys. Doklady 2, 416 (1958).

[23] J. Hubbard, Phys. Rev. Lett. 3, 77 (1959).

[24] D. Weingarten, Phys. Rev. Lett. 51, 1830 (1983).

[25] M. Lüscher, Commun. Math. Phys. 105, 153 (1986).

[26] P. F. Bedaque, H. W. Hammer, and U. van Kolck, Nucl. Phys. A676, 357 (2000), nuclth/9906032.

[27] Thanks to Jiunn-Wei Chen for discussions on this point.

[28] Thanks to Thomas Schaefer for suggesting the application of Lüscher's formula for the unbound case. 